\title{
Fuzzy Logic Controlled Semi-Active Floating Raft Vibration Isolation System
}

\author{
Amandeep Singh Ahuja*, Ashish Gupta \\ Department of Mechanical Engineering, Indian Institute of Technology Bombay, Powai, Mumbai - 400076 \\ *Corresponding Author: amannavy@sify.com
}

Copyright (C) 2014 Horizon Research Publishing All rights reserved.

\begin{abstract}
A warship may be detected in hostile waters because of its unique acoustic signature. A typical two-stage passive vibration isolation system used on ships and submarines to isolate onboard machinery is the floating raft isolation system. The passive isolation system, though robust, may result in substantially high transmission of forces to the foundation at certain excitation frequencies, adversely affecting the ship's stealth. This paper highlights the results of a simulation study aimed at the design of a semi-active floating raft vibration isolation system with the objective of mitigating the acoustic signature of a warship by minimising the transmission of forces, resulting from operation of onboard machinery, to the foundation. A semi-active control scheme with variable damping has been proposed for the floating raft, the variable damping being achieved by means of an electrorheological (ER) damper. A fuzzy logic controller has been designed to achieve the best isolation effect by analysing the characteristics of the frequencies in the excitation signal. The designed semi-active control system is subjected to a time-varying signal, each time-segment corresponding to a different optimal damping ratio. The MATLAB simulation results indicate that the proposed fuzzy logic control method is more effective in vibration isolation than the passive method thereby indicating the potential of the designed semi-active control system in reducing a ship's acoustic signature.
\end{abstract}

Keywords Floating Raft, Fuzzy Logic, Semi-active Control

\section{Introduction}

The term acoustic signature is used to describe a combination of acoustic emissions from ships and submarines. It is made up of a number of individual elements such as machinery noise, cavitation noise and hydrodynamic noise. Fig 1 depicts the various noise sources on ships and submarines.

The most obvious path for acoustic energy from onboard machinery to get into a ship's structure is through its attachment point to the ship's foundation. Vibrating equipment such as the main engines, generators, drive motor and numerous pieces of auxiliary equipment must be vibration-isolated from the hull. A typical passive vibration isolation system used in ships and submarines is the floating raft isolation system. The passive isolation system, though optimized for a range of excitation frequencies may, under certain conditions of excitation, result in substantially high transmission of forces to the foundation, adversely affecting the ship's stealth. Hence, there is a need to design a floating raft isolation system that will be able to adapt its characteristics depending on the features of the excitation signal at any given instant thereby providing the best isolation effect even if the excitation signal changes.

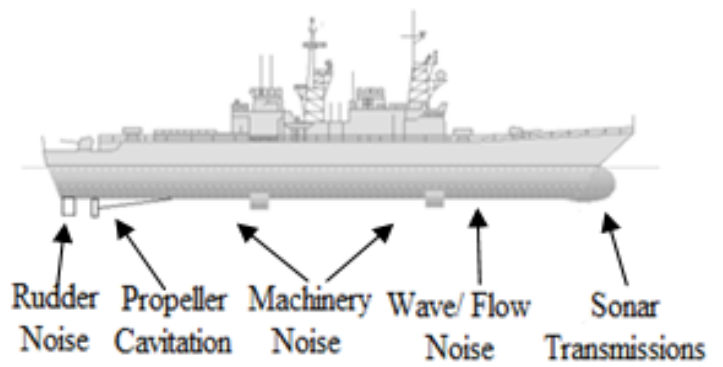

(a)

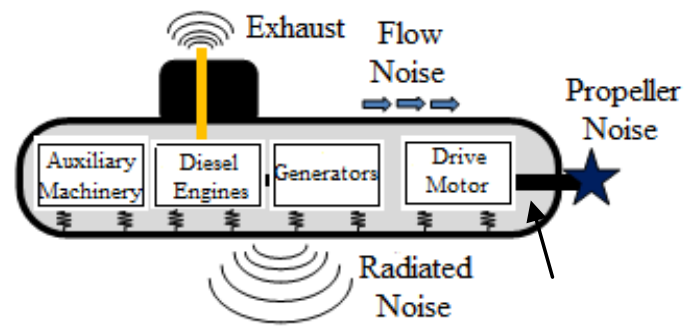

(b)

Figure 1. Noise sources on ships and submarines.

\section{Floating Raft Isolation System}

A floating raft isolation system consists of an intermediate mass, referred to as a raft, between an upper and lower set of vibration isolators. The machinery to be isolated is mounted on the raft. The objective of the semi-active vibration isolation system is to decrease the forces transmitted to the 
foundation. In the passive vibration isolation system, the objective is to minimise the vibration of the machine to be isolated. In the proposed semi-active system, the damping coefficient, $c$, can be varied by changing the voltage across the electrodes of an electrorheological damper. The structure of the semi-active floating raft vibration isolation systems is shown in Fig 2.

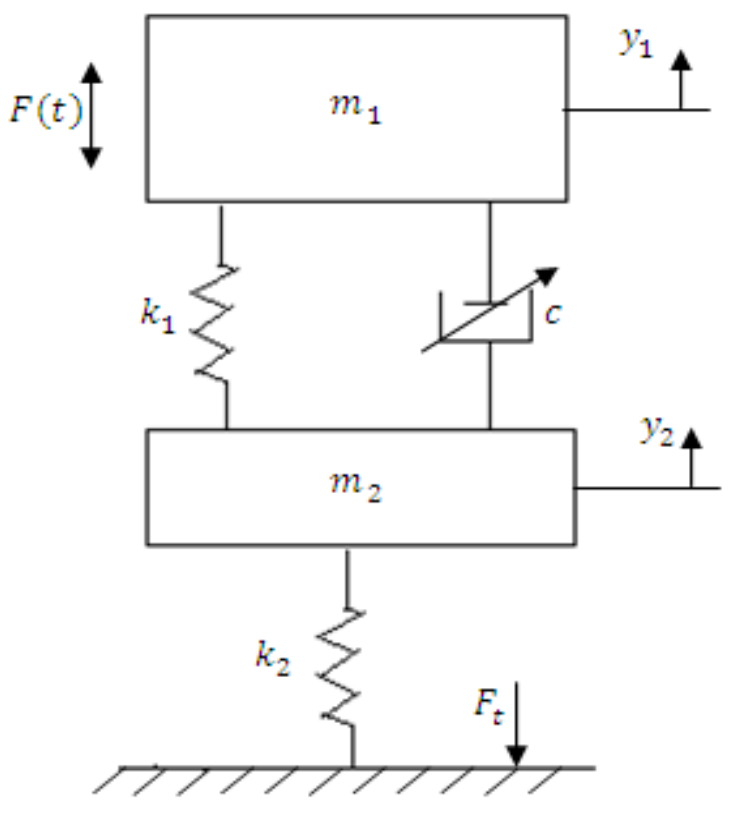

Figure 2. Semi-active vibration isolation system.

Two parameters have been defined to describe the capabilities of the two systems, the force transmission ratio in the semi-active system and the displacement transmission ratio in the passive system. It can be proven that these two parameters are naturally the same in one vibration isolation system. The force and displacement transmission ratios obtained by applying Laplace transforms to the equations of motion, assuming zero initial conditions, are as follows:

$$
\begin{gathered}
T_{f}(s)=\frac{F_{t}(s)}{F(s)}= \\
\frac{k_{2} c s+k_{1} k_{2}}{m_{1} m_{2} s^{4}+\left(m_{1}+m_{2}\right) c s^{3}+\left(m_{1} k_{1}+m_{1} k_{2}+m_{2} k_{1}\right) s^{2}+k_{2} c s+k_{1} k_{2}} \\
T_{d}(s)=\frac{Y_{1}(s)}{Y_{b}(s)}= \\
\frac{k_{2} c s+k_{1} k_{2}}{m_{1} m_{2} s^{4}+\left(m_{1}+m_{2}\right) c s^{3}+\left(m_{1} k_{1}+m_{1} k_{2}+m_{2} k_{1}\right) s^{2}+k_{2} c s+k_{1} k_{2}}
\end{gathered}
$$

It can be observed that the force transmission ratio, $T_{f}(s)$ and the displacement transmission ratio, $T_{d}(s)$ are equal. The parameters used by Sun et al. (2004) for their experimental set up shall be used for further analysis of the floating raft isolation system: $m_{1}=105.42 \mathrm{~kg}, m_{2}=59 \mathrm{~kg}$, $k_{1}=0.57 \mathrm{MN} / \mathrm{m}, k_{2}=1.9 \mathrm{MN} / \mathrm{m}$ and $c=1550 \mathrm{~N}-\mathrm{s} / \mathrm{m}$. Fig 3 shows the frequency response curves for the floating raft isolation system with the above parameters. The three curves correspond to damping ratios, $\xi=0.01, \xi=0.5$ and $\xi=1$. The frequency response curves for various damping ratios are observed to pass through a fixed point corresponding to an inter-cross frequency, $f_{c}=12.5 \mathrm{~Hz}$.

Dhenesh Manik (2012) explained that the two natural frequencies of the isolation system can be determined from the frequency equation,

$$
\left|K-\omega^{2} M\right|=0
$$

From Eq. (3), the first natural frequency is determined to be $63.46 \mathrm{rad} / \mathrm{s}$ or $10.1 \mathrm{~Hz}$ while the second natural frequency is $207.97 \mathrm{rad} / \mathrm{s}$ or $33.1 \mathrm{~Hz}$.

\section{Optimal Damping Ratio}

For further analysis, only the first natural frequency, represented by $f_{o}$, has been considered because in most cases the vibration isolation system operates in the low frequency band rather than in the high frequency band. It can be observed from Fig 3 that the inter-cross frequency, $f_{c}$ lies between the first and second natural frequencies. When the primary mass is excited by a signal consisting of a single dominant frequency, the damping ratio that results in a minimal transmission ratio can be observed graphically from Fig 3. However, when the excitation signal is composed of multiple frequencies of different amplitudes, the optimal damping ratio cannot be directly obtained and requires further analysis.

\subsection{Excitation Signal with a Single Frequency}

For an excitation signal with a single main frequency, the optimal damping ratio that results in the best isolation effect can directly be obtained from Fig 3 . When the main frequency is lower than the inter-cross frequency, the maximal damping ratio, $\xi_{\max }$ can be chosen as $\xi_{\text {opt }}$, and the minimal damping ratio, $\xi_{\min }$ can be applied when the main frequency is higher than $f_{c}$. Fig 3 shows that, for the force transmission ratio to be minimum, the damping ratio must ideally be equal to 1 if the excitation frequency is lesser than $f_{c}$, and must be equal to 0.1 if the excitation frequency is greater than $f_{c}$.

\subsection{Complex Excitation Signal}

In reality, the excitation signal may be a complex combination of multiple frequencies and the semi-active system must be designed to adopt the damping ratio that results in a minimal transmission ratio. For such a complex input signal, we cannot use the above method to achieve the best control effect because the signal has several frequencies with different amplitudes. A best damping ratio, cannot be obtained readily in that some frequencies are lower than $f_{c}$ while others are higher with different amplitudes. In fact, many machines work together when the ship sails and the excitation signal to the floating raft isolation system contains several frequencies at the same time. Further, the excitation signal to the floating raft isolation system may change with time, depending on the machines in operation at a given instant. In order to solve this problem, a fuzzy logic controller has been designed to select the best damping ratio, according to the features of the complex input signal. 


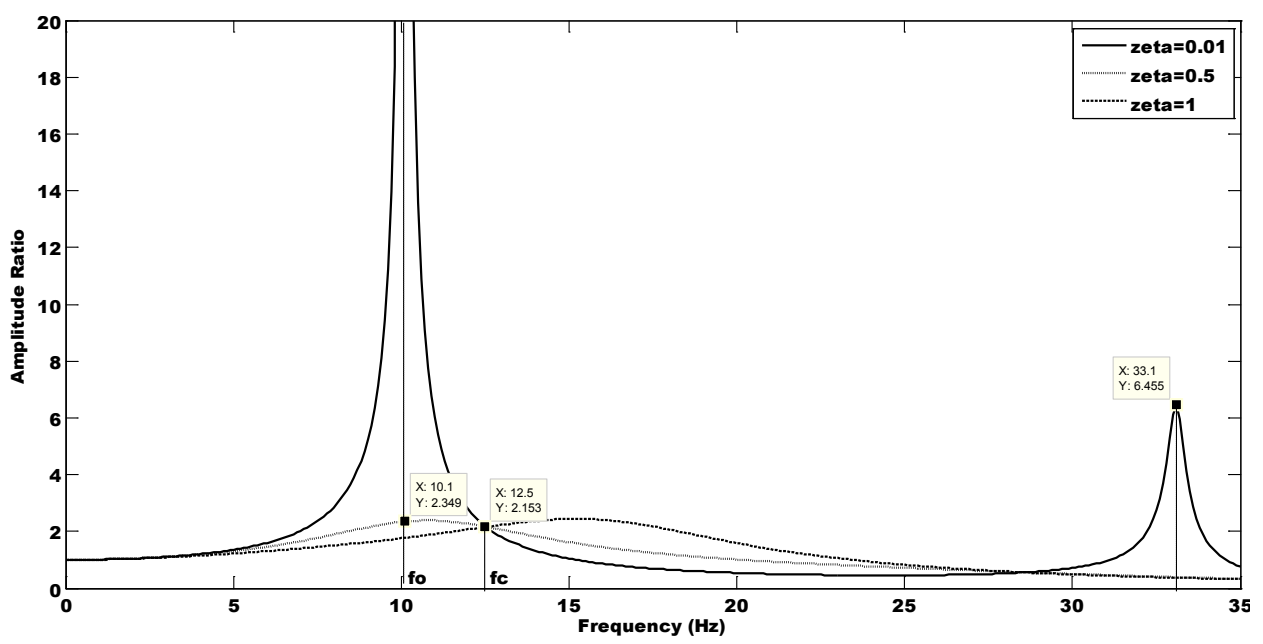

Figure 3. Transmission ratio for various damping ratios.

\section{Fuzzy Logic Controller}

The input variables to the fuzzy logic controller are the position coefficient $\left(F_{1 i}\right)$ and the amplitude coefficient $\left(A_{1 i}\right)$ while the output is the effect coefficient $\left(\beta_{i}\right)$ of a given frequency in the excitation signal. The weighting coefficient, $\beta_{1 i}$, of each frequency is determined from the effect coefficient. The input coefficients are defined as follows:

$$
F_{1 i}=\left\{\begin{array}{c}
\left|f_{1}-f_{0}\right| /\left|f_{i}-f_{0}\right|, f_{i} \neq f_{0} \\
5, f_{i}=f_{0}
\end{array} \quad \mathrm{i}=2,3, \ldots \ldots, \mathrm{n}\right.
$$

where $f_{0}$ is the first natural frequency; $f_{1}$ is the frequency whose amplitude is the largest and $f_{i}(i=2,3, \ldots ., n)$ stands for the other frequencies in the excitation signal.

$$
A_{1 i}=A_{1} / A_{i}
$$

The memberships functions for $F_{1 i}, A_{1 i}$ and $\beta_{i}$ are shown in Fig. 4(a), 4(b) and 4(c) respectively.

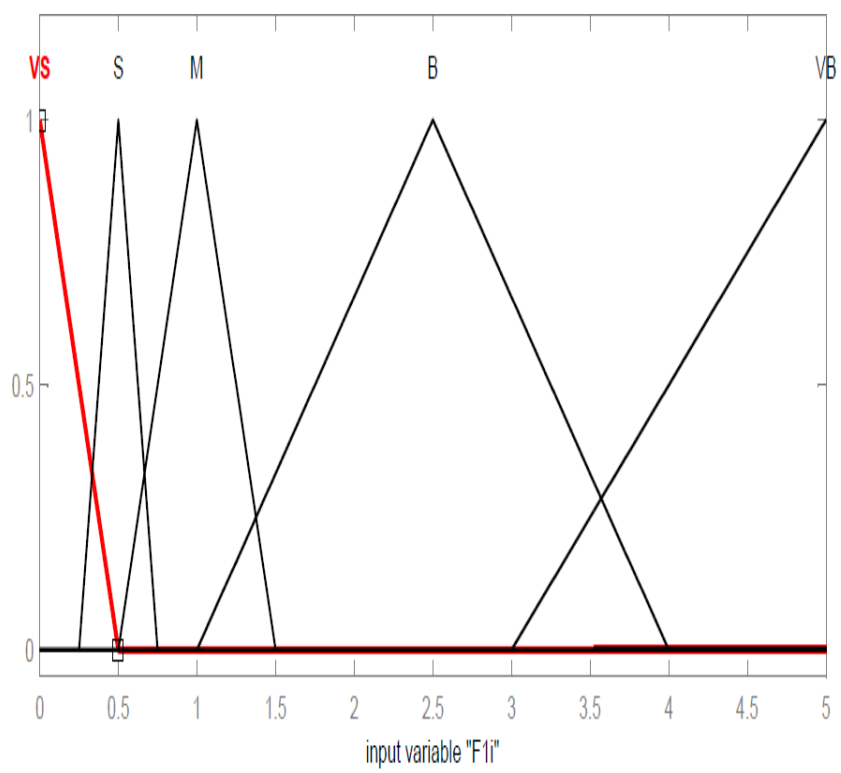

(a)

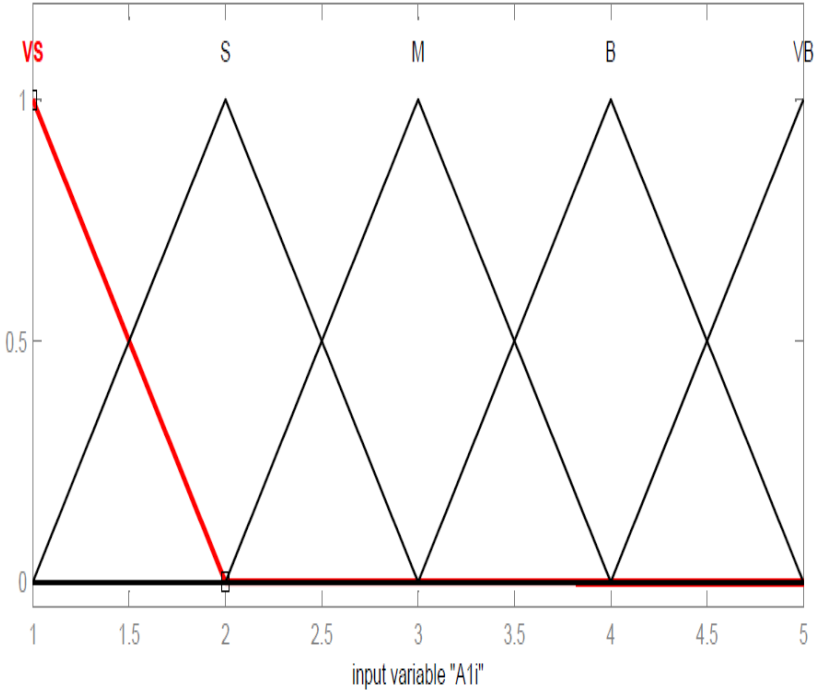

(b)

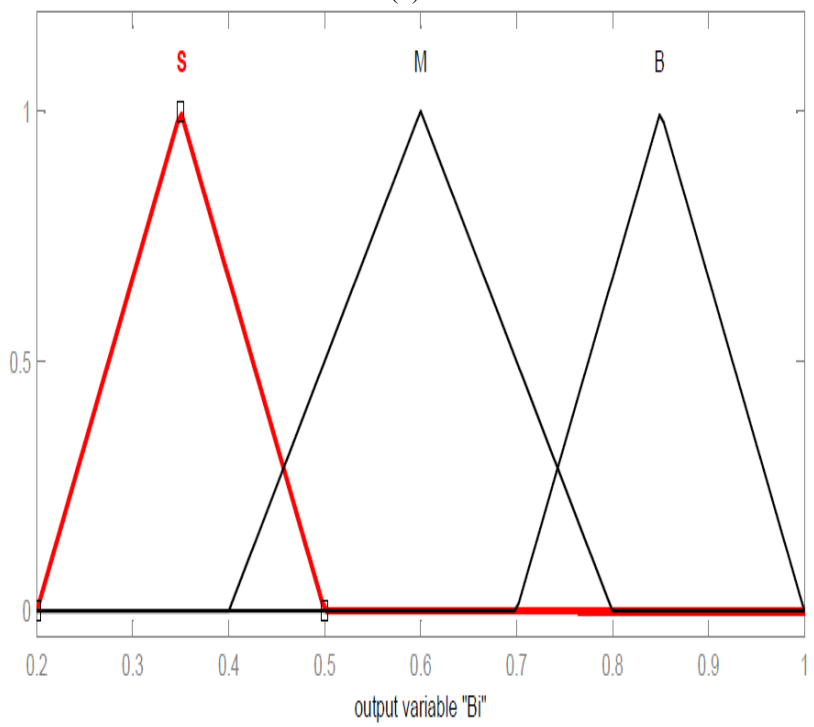

(c)

Figure 4. Membership functions for the input and output coefficients. 
Rao et al. (1995) explained that the fuzzy logic controller makes decisions according to control rules and selects the best effect coefficient for each frequency. The defuzzification method employed is the centroid of area method.

Table 1. Rule base for the fuzzy logic controller

\begin{tabular}{|c|c|c|c|c|c|c|}
\hline \multicolumn{7}{|c|}{$\begin{array}{c}\beta_{i} \text { Position Coefficient } \\
\left(F_{1 i}\right)\end{array}$} \\
\hline \multirow{6}{*}{$\begin{array}{c}\text { Amplitude } \\
\text { Coefficient } \\
\left(A_{1 i}\right)\end{array}$} & & VS & $\mathrm{S}$ & $\mathrm{M}$ & B & $\mathrm{VB}$ \\
\hline & VS & $\mathrm{M}$ & $\mathrm{M}$ & B & B & B \\
\hline & $\mathrm{S}$ & $\mathrm{S}$ & $\mathrm{M}$ & B & B & B \\
\hline & $\mathrm{M}$ & $\mathrm{S}$ & $\mathrm{S}$ & $\mathrm{M}$ & $\mathrm{M}$ & B \\
\hline & B & $\mathrm{S}$ & $\mathrm{S}$ & $\mathrm{M}$ & $\mathrm{M}$ & B \\
\hline & VB & $\mathrm{S}$ & $\mathrm{S}$ & $\mathrm{S}$ & $\mathrm{M}$ & B \\
\hline
\end{tabular}

The following equation is built to find the optimal damping ratio of the ER damper:

$$
Y(\xi)=A_{1} G\left(f_{1}, \xi\right)+\sum_{2}^{n} \beta_{1 i} A_{i} G\left(f_{i}, \xi\right) \mathrm{i}=2,3, \ldots \ldots, \mathrm{n}
$$

where $s_{i}$ is the complex variable defined as $s_{i}=j 2 \pi f_{i}$; $G\left(f_{i}, \xi\right)$ is the modulus of the system transfer function at $f_{i}$ and is a function of the damping ratio, $\xi$. The weighting coefficient, $\beta_{1 i}$ in Eq. (6) can be determined from the following equation:

$$
\beta_{1 i}=\beta_{i} /\left(1-\beta_{i}\right), \mathrm{i}=2,3,
$$

The optimal damping ratio, $\xi_{\text {opt }}$ at a given frequency or a set of frequencies corresponds to a minimum value of $Y$ in Eq. 6.

\section{Complex Input Signal}

The excitation signal at any instant of time depends on the machines in operation at that instant. Moreover, the operating speeds of the machines are subject to change. Thus, the floating raft may be subjected to excitation signals that change continually with time. Consider a floating raft isolation system subjected to a complex excitation signal that changes over three time segments during a simulation time of 1 second. Fig 5 shows the input signal to the floating raft where the simulation time is expressed in milliseconds. The excitation signal in each time segment is defined in Table 2.

Table 2. Excitation signal in the three time segments.

\begin{tabular}{|c|c|c|c|}
\hline S1 No & Time Segment & Excitation Signal & $\xi_{\text {opt }}$ \\
\hline 1 & $0-0.3 \mathrm{~s}$ & $\mathrm{~F}(\mathrm{t})=1000 \sin (2 \mathrm{n} 8 \mathrm{t})$ & 1 \\
\hline 2 & $0.3-0.7 \mathrm{~s}$ & $\begin{array}{c}\mathrm{F}(\mathrm{t})=1000 \sin (2 \Pi 8 \mathrm{t})+850 \\
\sin (2 \Pi 16 \mathrm{t})\end{array}$ & 0.4 \\
\hline 3 & $0.7-1.0 \mathrm{~s}$ & $\begin{array}{c}\mathrm{F}(\mathrm{t})=1000 \sin (2 \Pi 10 \mathrm{t})+900 \\
\sin (2 \pi 14 \mathrm{t})\end{array}$ & 0.6 \\
\hline
\end{tabular}

\subsection{Transfer Function Method}

Each of the excitation signals in Table 2 has a corresponding optimum damping ratio. If a different excitation signal is considered, the optimum damping ratio corresponding to that signal would first have to be determined. The lsim command in MATLAB allows the output of a transfer function to be obtained for a given input signal and has been utilised in the MATLAB code for comparing the passive and semi-active systems. The transfer function for the displacement of the intermediate mass is represented as follows:

$\frac{Y_{2}(s)}{F(s)}=\frac{c s+k_{1}}{m_{1} m_{2} s^{4}+\left(m_{1}+m_{2}\right) c s^{3}+\left(m_{1} k_{1}+m_{1} k_{2}+m_{2} k_{1}\right) s^{2}+k_{2} c s+k_{1} k_{2}}(8)$

The displacement response of the intermediate mass, obtained by utilising the above equations in a MATLAB code, and a comparison of the force transmitted to the foundation are shown in Figures 6 and 7 respectively. The response of the passive system has been represented by a dashed curve while the semi-active system response is given by a solid curve in each figure.

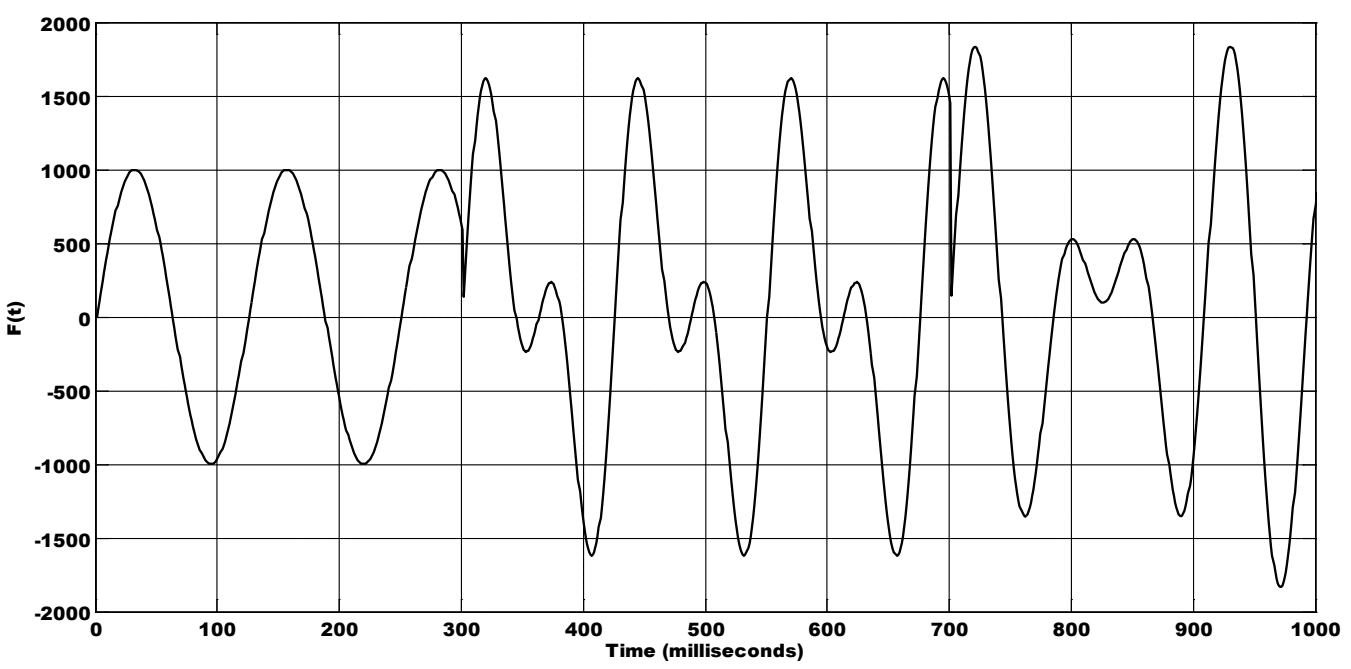

Figure 5. Input signal to the floating raft. 


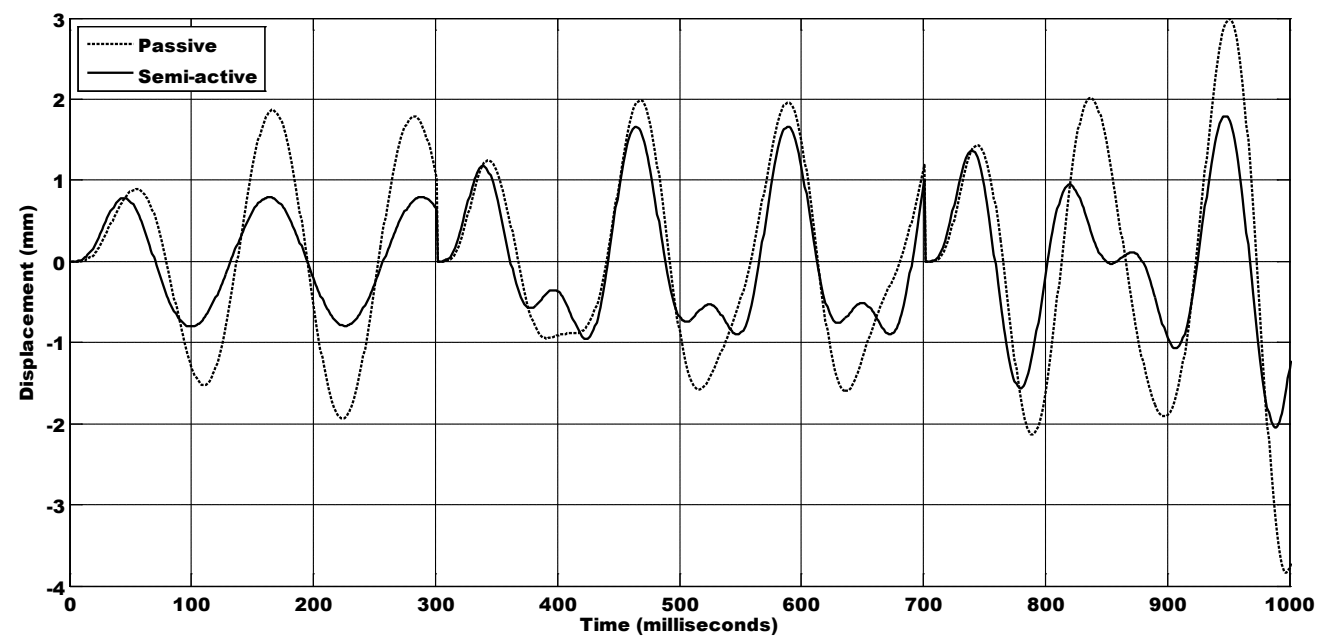

Figure 6. Intermediate mass displacement response.

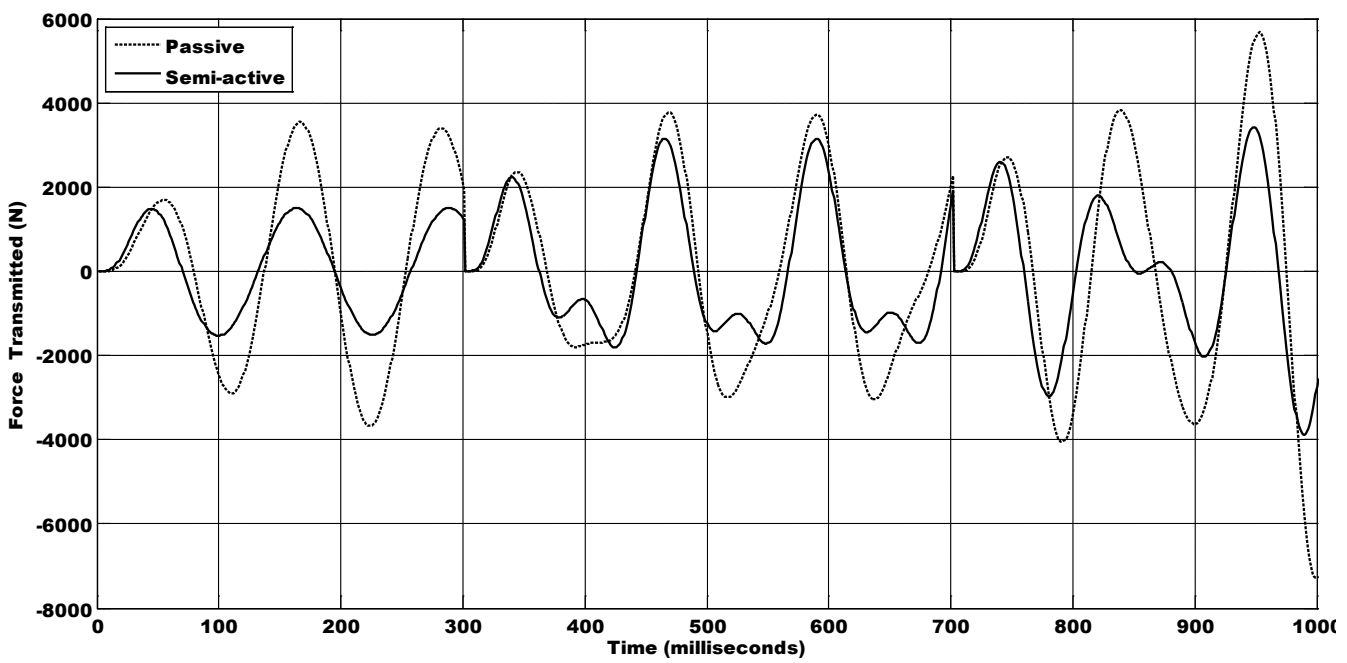

Figure 7. Variation of force transmitted to the foundation.

\section{Conclusion}

It can be observed from Fig 6 that the displacement response of the intermediate mass is reduced over the total simulation time in case of the semi-active system. Fig 7 shows a significant reduction in the force transmitted to the foundation over the total simulation time. Since force is transmitted to the foundation through the spring connecting the intermediate mass to the foundation, there is also a corresponding reduction in the force transmitted to the foundation over the complete simulation period of 1 second. This indicates the potential of the designed fuzzy logic control system in reducing the acoustic signature of ships and submarines.

\section{REFERENCES}

[1] Howard, C.Q. (2010), "Technologies for the Management of the Acoustic Signature of a Submarine", School of Mechanical Engineering, The University of Adelaide,
Adelaide, Australia University of Adelaide, Australia, 10 Nov

[2] Howard, CQ. (2011), "A New Cost Function Algorithm for Adaptive-passive Vibration and Acoustic Controllers" in Proceedings of Acoustics 2011, Annual conference of the Australian Acoustical Society, Gold Coast, Queensland

[3] Niu et al. (2005), "On Active Vibration Isolation of Floating Raft System", School of Mechanical Engineering, Shandong University, People's Republic of China.

[4] Zhao et al. (2007), "Fuzzy-PID Control Method for Two-Stage Vibration Isolation System", Institute of Noise and Vibration, Naval University of Engineering, Wuhan, China

[5] Symans et al. (1999), "Semi-active Control Systems for Seismic Protection of Structures: A State-of-the-art Review", Department of Civil and Environmental Engineering, Washington State University, Pullman, WA 99164, USA

[6] Rao et al. (1995), "A Tunable Fuzzy Logic Controller for Vehicle Active Suspension Systems", Department of Electrical Engineering, Indian Institute of Technology, Madras, 600036, India

[7] Sun et al. (2004), "Signal Frequency-based Semi-active Fuzzy Control for Two-stage Vibration Isolation System", 
Department of Information Measurement Technology and Instruments, Shanghai Jiaotong University, Shanghai, 2000030, PR China

[8] Dhanesh Manik (2012), Control Systems, First Edition, Cenage Learning India

[9] Martin et al. (1998), "Structure and Dynamics of Electrorheological Fluids", Advanced Materials Physics Division, Sandia National Laboratories, Albuquerque, New Mexico 87185-1421
[10] Park \& Gyu (2003), "Vibration Simulation using MATLAB”, Department of Precision Engineering, Kyoto University, Kyoto, Japan

[11] Tian et al. (2008), "Vibration Control of Ship Structures", School of Engineering Systems, Queensland University of Technology, Brisbane, Australia

[12] Xun et al. (2002), "Active Vibration Control of an Intermediate Mass", School of Mechanical Engineering, University of Adelaide, Adelaide, Australia (13 November) 